\title{
Absence of evidence is not evidence of absence: Nanopore sequencing and complete assembly of the European lobster (Homarus gammarus) mitogenome uncovers the missing nad2 and a new major gene cluster duplication
}

\author{
Han Ming Gan ${ }^{1,2,3,4^{*}}$ D, Frederic Grandjean ${ }^{5}$, Tom L. Jenkins ${ }^{6}$ and Christopher Mervyn Austin ${ }^{1,2,3,4}$
}

\begin{abstract}
Background: The recently published complete mitogenome of the European lobster (Homarus gammarus) that was generated using long-range PCR exhibits unusual gene composition (missing nad2) and gene rearrangements among decapod crustaceans with strong implications in crustacean phylogenetics. Such atypical mitochondrial features will benefit greatly from validation with emerging long read sequencing technologies such as Oxford Nanopore that can more accurately identify structural variation.

Results: We re-sequenced the H. gammarus mitogenome on an Oxford Nanopore Minion flowcell and performed a long-read only assembly, generating a complete mitogenome assembly for $\mathrm{H}$. gammarus. In contrast to previous reporting, we found an intact mitochondrial nad2 gene in the $\mathrm{H}$. gammarus mitogenome and showed that its gene organization is broadly similar to that of the American lobster ( $H$. americanus) except for the presence of a large tandemly duplicated region with evidence of pseudogenization in one of each duplicated protein-coding genes.

Conclusions: Using the European lobster as an example, we demonstrate the value of Oxford Nanopore long read technology in resolving problematic mitogenome assemblies. The increasing accessibility of Oxford Nanopore technology will make it an attractive and useful tool for evolutionary biologists to verify new and existing unusual mitochondrial gene rearrangements recovered using first and second generation sequencing technologies, particularly those used to make phylogenetic inferences of evolutionary scenarios.
\end{abstract}

Keywords: Homarus gammarus, Mitogenome, Nanopore sequencing, Lobster, Tandem duplication, PCR bias

\section{Background}

The European lobster (Homarus gammarus (Linnaeus, 1758)) is an economically important crustacean species with a broad geographic range across the eastern Atlantic Ocean, extending from the Arctic Circle to Morocco [1].The only other species placed in the genus Homarus

\footnotetext{
* Correspondence: han.gan@deakin.edu.au

${ }^{1}$ Deakin Genomics Centre, Deakin University, Geelong, VIC 3220, Australia

${ }^{2}$ Centre for Integrative Ecology, School of Life and Environmental Sciences, Deakin University, Geelong, VIC 3220, Australia

Full list of author information is available at the end of the article
}

is the closely related American lobster (Homarus americanus (Fabricius, 1775)) with natural range on the opposite side of the North Atlantic [2]. Despite its socio-economic importance, early population genetics of the clawed lobsters employing various genetic markers such as mtDNA, microsatellites and random amplified polymorphic DNA focused on the American lobster. It is only in the last two decades that the genetic diversity and population structure of $H$. gammarus have been actively investigated using molecular genetic techniques [3-6]. One of the most comprehensive genetic

(c) The Author(s). 2019 Open Access This article is distributed under the terms of the Creative Commons Attribution 4.0 International License (http://creativecommons.org/licenses/by/4.0/), which permits unrestricted use, distribution, and 
investigations of Homarus gammarus was by Triantafyllidis and co-workers who sampled 3283 individuals from 44 populations across its native geographical distribution and analyzed the diversity and distribution of restriction fragment length polymorphism of a 3-kb mitochondrial DNA amplicon [7].

The complete mitogenome of $H$. americanus has been sequenced, assembled and annotated and is consistent with the pancrustacean gene order [8]. However, in stark contradistinction, the recently published $H$. gammarus mitogenome [9] deviates substantially as it was reported to have not only a large inversion but to be also missing the $n a d 2$ genes and a few tRNA genes [10]. While minor and major departures from the pancrustacean gene order have been observed in multiple lineages of decapod crustaceans notably among scampi and Southern Hemisphere freshwater crayfish species [11-16], all 13 mitochondrial protein-coding genes are always present in the assembled mitogenome occasionally with some species having duplicated tRNA genes and occassionally the control region [14]. The finding of mitogenome structural variations is of interest as molecular synapomorphies and for providing insights in mitogenome evolution and it is quite rare to find such significant mitogenome rearrangements and missing genes between closely related species. A necessary caveat, however, is the assumption that mitogenome studies reporting rearrangements and missing genes have used appropriate wet lab and bioinformatics protocols that are robust to potential errors or misinterpretations. It is thus always beneficial that such atypical findings be verified by other laboratories.

Traditionally, complete mitogenomes have been recovered using long-range PCR followed by multiple Sanger sequencing reactions (primer walking) using overlapping fragments to produce a complete assembly. One of the assumptions of this approach is that the mitogenome of interest has a gene order that is identical to, or at least highly similar to the reference sequence. In other words, species with mitogenomes with substantial gene rearrangement relative to an appropriate reference mitogenome (usually a related species) will present a significant challenge to the complete recovery of mitogenomes using PCR-based approach as this will generally lead to failed PCR reactions, amplicon of unanticipated lengths and multiple amplicons as well as the potential amplification of nuclear-derived mtDNAs [17-19]. The advent of high-throughput short-read sequencing technologies has greatly enhanced the accuracy and efficiency of mitogenome recovery by allowing mitogenomes to be sequenced without prior sequence knowledge or gene order [20,21]. By sequencing multiple small segments of the DNA ( 100-250 bp) in parallel, such an approach essentially removes the assumptions and limitations of long-range PCR and also allows the recovery of mitogenomes from degraded DNA samples including those from museum specimens [22, 23]. Nevertheless, a major pitfall of this technology is the inability of short reads to span highly repetitive regions that exist in some mitogenomes particularly in the control region. This means that assemblies may give a fragmented mitogenome that precludes the accurate inference of gene order organization, although gap closing using custom design primers and Sanger sequencing can be successful in resolving such incomplete assemblies [24].

Three common approaches are used for the assembly of mitogenome from short read data e.g. whole mitogenome reference mapping, bait-and-extend from a single gene and de novo assembly; yet, these approaches can lead to different outcomes when applied to the same dataset [25]. Whole mitogenome reference mapping usually works well when the sequences are obtained from individuals from the same or a closely related species. However, some caution is advised in assembling mitogenomes in this way as even congeneric species can show variations in mitochondrial gene order as recently reported in the Southern Hemisphere burrowing freshwater crayfish, Engaeus spp. [14]. Inferring novel mitochondrial gene organization is generally more robust using either de novo assembly or single gene baiting approach as they do not make assumptions on a prior gene order information. Failure to recover a complete circular mitogenome is usually associated with anomalies in the sequence composition ranging from an overly repetitive control region to large-scale tandem duplications that will require gap-closing via PCR which is not always successful.

The recent improvements in Oxford Nanopore technology most notably in its read accuracy, flow cell stability, and sequencing algorithm have truly democratized long-read sequencing, providing individual researchers with sequencing capacity that is comparable to those previously found only in large sequencing centers [26]. To date, several studies have explored combining accurate Illumina reads with long $(>1 \mathrm{~kb})$ but more error-prone Nanopore reads to improve whole genome assemblies [27-29]. More recently, this approach has been used to assemble the complete mitogenome $(\sim 16 \mathrm{~kb})$ of the economically important green-lipped mussel [30]. However, the mitogenome exhibits the typical 13 protein-coding genes, 22 tRNAs, 2 rRNAs and a control region, suggesting that Illumina short read data alone can also accomplish the same objective as reported in various mussel mitogenomic studies [31-34]. Unlike the green-lipped mussel mitogenome, the Homarus gammarus mitogenome represents an interesting case due to its atypical gene organization that has been partially verified using PCR across a few individuals in a follow-up study [10]. Furthermore, the high genetic variation observed in the $3 \mathrm{~kb}$ mitochondrial region that excludes the control region is unexpected [7] but can be explained by the presence of a large tandem duplication event followed by pseudogenization which warrants further investigation. In this study, we 
generated the first Illumina dataset for Homarus gammarus providing strong evidence for structural incongruence with the published $H$. gammarus mitogenome. By fully exploiting the ability of Nanopore long-read to span large repeats, we were able to circularize the mitogenome leading to the discovery of the large tandemly duplicated mitogenome region with strong evidence of pseudogenization and the presence of the purportedly missing nad2 gene.

\section{Methods}

\section{Sample collection and gDNA extraction}

A wild-caught European lobster from the Atlantic Ocean was purchased from a commercial fisherman. Approximately $500 \mathrm{mg}$ of tail muscle tissue was dissected and preserved in 95\% ethanol. DNA extraction was performed using an SDS- based lysis method followed by chloroform purification as previously described [35]. To confirm mitogenome features, notably the presence of nad2 gene, additional European lobsters were collected from five different and distant European regions (Fig. 2c) as previously described [36].

\section{Illumina sequencing}

One hundred ng of gDNA was sonicated to a fragment size of $500 \mathrm{bp}$ using a Covaris ultrasonicator and processed using NEB Ultra DNA library prep kit according to the manufacturer's instructions. The constructed library was quantified and size-estimated using Qubit and Tapestation2100, respectively. Sequencing of the library was performed on an Illumina MiSeq located at the Monash University Malaysia Genomics Facility using the run configuration $2 \times 250 \mathrm{bp}$.

\section{Nanopore sequencing}

Two $\mu \mathrm{g}$ of gDNA was repaired using NEB FFPE Repair Mix and subsequently processed using the Nanopore LSK108 kit according to the manufacturer's instructions. The library (premixed with loading beads) was pipetted into a previously used and washed FLO-MIN106 flow cell. The flow cell was fixed to a MINION device and sequencing was performed for $16 \mathrm{~h}$ (overnight) on a Linux Ubuntu desktop through the MinKnow software version 2.0.

\section{Mitogenome assembly and annotation}

Illumina-only mitogenome assembly was performed using two reference-based assemblers (MITObim and NOVOplasty) and a de novo assembler (Megahit) [37-39]. For MITObim, 3 separate assemblies were performed using different mitochondrial gene baits ( $n a d 2,16 \mathrm{~S}$ rRNA, and $c y t b$ ) from the American Lobster, $H$. americanus (GenBank accession number: NC_015607). Completeness and the presence of anomalies in the assembled mitogenome were determined by conducting BLASTX search of the contig against the 13 mitochondrial proteins from $H$. americanus. For long-read only assembly, Nanopore long-reads were first aligned to the mitogenomes of $H$. americanus and $H$. gammarus. The aligned reads were error-corrected, trimmed and assembled using CANU v1.7 [40]. The graphical fragment assembly of contig flagged as circular by CANU was visualized on Bandage v0.8.1 [41]. A self-against-self nucleotide similarity search of the assembled contig was performed and visualized with EasyFig v2.2.2 [42] to confirm the presence of highly similar flanking regions in the contig. Polishing of the CANU assembly used Unicycler_Polish module in Unicycler v0.4.4 that mapped Illumina reads to the raw assembly using Bowtie2 (--very-sensitive-local) and piped the alignment file into Pilon v1.22 to specifically correct for small indel and SNP [43-45]. Circularization of the polished mitogenome and re-orientation of the contig 5 ' end to cox 1 gene used Circules.py python script that is part of the MITObim v1.9 software [39]. The circularized mitogenome was polished again to fix any remaining assembly errors in sequence previously originating from the flanking regions of the uncircularized contig. These errors may have been missed in the first polishing step due to the repetitiveness and lower read coverage in the flanking regions. The polished mitogenome was annotated using the MITOS webserver [46] and each protein-coding gene was manually inspected with their start and stop position adjusted when necessary using the $H$. americanus mitogenome as the reference. The polished $H$. gammarus mitogenome was subsequently used as the reference to reconstruct the mitogenomes of the 5 additional $H$. gammarus individuals through MITObim v1.9 “-quick" option.

\section{Visualization of mitochondrial gene organization and alignment}

$\mathrm{Nad} 2$ proteins were aligned and visualized with MAFFT v7.123b and ESPript v3.0, respectively [47, 48]. Maximum likelihood tree based on the amino alignment was inferred using IQ-TREE v1.65 with 1000 ultra-fast bootstrap replicates [49]. Circular comparisons between the newly assembled $H$. gammarus mitogenomes used BRIG v0.95 [50] while linear comparison of gene organization between Homarus spp. used EasyFig v2.2.2 [42]. Alignment of Illumina and Nanopore reads to the complete mitogenome was performed using bowtie 2 v2.3.0 and minimap2 v2.10-r761, respectively and visualized in IGV v2.4.5 $[51,52]$. GC plot of the mitogenome (windowsize = $500 \mathrm{bp)}$ was constructed in Artemis v17.0.1 [53]. Nucleotide and amino acid alignment of pseudogenes used MACSE v2.01 (gc_code =5) followed by alignment visualization in Jalview $[54,55]$.

\section{Results}

The mitochondrial NADH dehydrogenase subunit 2 (nad2) gene has not been lost in Homarus gammarus

A total of 6,029,826 paired-end reads $(\sim 1.5$ gigabases, SRA accession:SRP157954) were generated from MiSeq 
sequencing. Such a sequencing output is typically sufficient for the reconstruction of mitogenome via reference mapping or de novo assembly approach. Contrary to the previously reported loss of nad2 in H. gammarus [9] (Fig. 2a), mitogenome reconstruction using $H$. americanus nad2 as the bait generated a contig which spans the entire nad2 gene of $H$. gammarus in addition to other bona fide mitochondrial genes in its vicinity with. Most contig extensions occurring at the 3 ' end of the bait (Table 1), suggesting a difficult-to-assemble region possibly in the form of long repeats upstream of the nad2 gene. The translated ORF of the assembled nad2 shows high pairwise amino acid identity (96.7\%) to that of $H$. americanus with only 11 amino acid mismatches out of 333 aligned protein length (Fig. 1a). Protein domains associated with NADH dehydrogenase subunit 2 are present in the translated ORF (Fig. 1b), suggesting a similar function. Maximum likelihood tree construction based on amino acid alignment provides phylogenetic support for its annotation as nad2 given its well-supported monophyletic clustering ( $88 \%$ ultrafast bootstrap support) with the nad2 protein of H. americanus (Fig. 1c).

\section{Illumina-based genome skimming approach fails to generate the complete Homarus gammarus mitogenome assembly}

Both reference mapping (single-gene bait) and de novo assembly based on Illumina-only short reads failed to generate contigs that are longer than $15 \mathrm{~kb}$ or contain flanking identical region typically required to infer mitogenome circularity. The longest MITObim assembly was achieved using the $H$. americanus cob gene as the initial read bait with a final assembly length of $14 \mathrm{~kb}$ covering all 13 PCGs (Table 1). On the other hand, BlastX search against the de novo-assembled contigs identified multiple small mitochondrial contigs, some of which exhibit significant homology to one another. For example, ctg154136 (5 kb) and ctg252123 (4 kb) from the MEGAHIT assembly harbor a nearly similar set of PCGs although the some of the PCGs in the latter contig are likely pseudogenes.

\section{Nanopore long-read assembly confirms mitogenome circularity}

An overnight run of a used nanopore flow cell generated 40,687 reads with an accumulated length of 100 megabases and N50 of $2811 \mathrm{bp}$. The basecalled fastq files were archived in Zenodo (https://doi.org/10.5281/zenodo. 1345356). Approximately $0.19 \%$ of the sequencing reads were mapped to the $H$. americanus and $H$. gammarus mitogenomes (255 kb, N50 of $4639 \mathrm{bp}$ ). De novo assembly of the mapped long-reads generated a $26 \mathrm{~kb}$ contig suggested to be "circular" by CANU. This was subsequently verified by the circular representation of the assembly graph in Bandage (Additional file 1: Figure S1A). Self-to-self nucleotide alignment of the contig indicates that up to $6 \mathrm{~kb}$ of its flanking regions exhibit strikingly high similarity, thus providing additional evidence for mitogenome circularity (Additional file 1: Figure S1B). Four rounds of Pilon polishing identified and corrected 402 indels/SNPs in the initial CANU-assembled contig. Subsequent polishing of the circularized and re-oriented contig corrected an additional 5 indels/SNPs, generating a final, complete and circular mitogenome with a total length of 20,303 bp and 31.68\% GC-content. MITOS annotation confirmed the presence of the typical 13 PCG but also identified 6 duplicated PCGs some of which contained internal stop codons and/or frameshift mutation. The final polished and annotated mitogenome assembly has been deposited in GenBank under the accession number MH747083.

\section{Consistent recovery of nad2 in $\mathrm{H}$. gammarus individuals from different populations}

Linear comparison of the recently published H. gammarus complete mitogenome (GenBank: NC_020020) with our new assembly revealed three substantial structural variations namely a tandem duplication involving

Table 1 Performance of different assemblers in the recovery of Homarus gammarus mitogenome

\begin{tabular}{|c|c|c|}
\hline Assembler & $\begin{array}{l}\text { Contig Length } \\
\text { (bait/contiglD) }\end{array}$ & Protein coding gene (number of intact genes) \\
\hline \multirow{3}{*}{$\begin{array}{l}\text { MITObim } \\
\text { v. } 1.9\end{array}$} & $14,885 \mathrm{bp}(\mathrm{rrnL})$ & nad1, cytb, nad6, nad4L, nad4, nad5, nad3, cox3, atp6, atp8, cox2, cox1, nad2 (13) \\
\hline & 13,143 bp (cytb) & nad2, cox1, cox2, atp8, atp6, cox3, nad3, nad5, nad4, nad4L, nad6, cytb, nad1 (13) \\
\hline & 6984 bp (nad2) & nad2, cox1, cox2, atp8, atp6, cox3, nad3, nad5, cox2 (9) \\
\hline \multirow{3}{*}{$\begin{array}{l}\text { Megahit } \\
\text { v1.1.2 }\end{array}$} & 5162 bp (ctg154136) & nad2, cox1, cox2, atp8, atp6, cox3 (6) \\
\hline & 4045 bp (ctg252123) & $\operatorname{cox} 3^{a}, \operatorname{atp} 6, \operatorname{atp} 8, \operatorname{cox} 2^{a}, \operatorname{nad} 5^{b}, \operatorname{nad} 3, \operatorname{cox} 3$ (4) \\
\hline & 10,025 bp (ctg253367) & nad1, cytb, nad6, nad4L, nad4, nad5, nad3, cox3 (8) \\
\hline $\begin{array}{l}\text { NOVOplasty } \\
\text { v2.7.1 }\end{array}$ & $22,032 \mathrm{bp}$ & 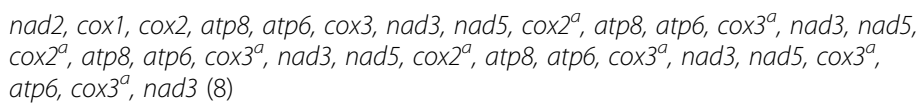 \\
\hline
\end{tabular}




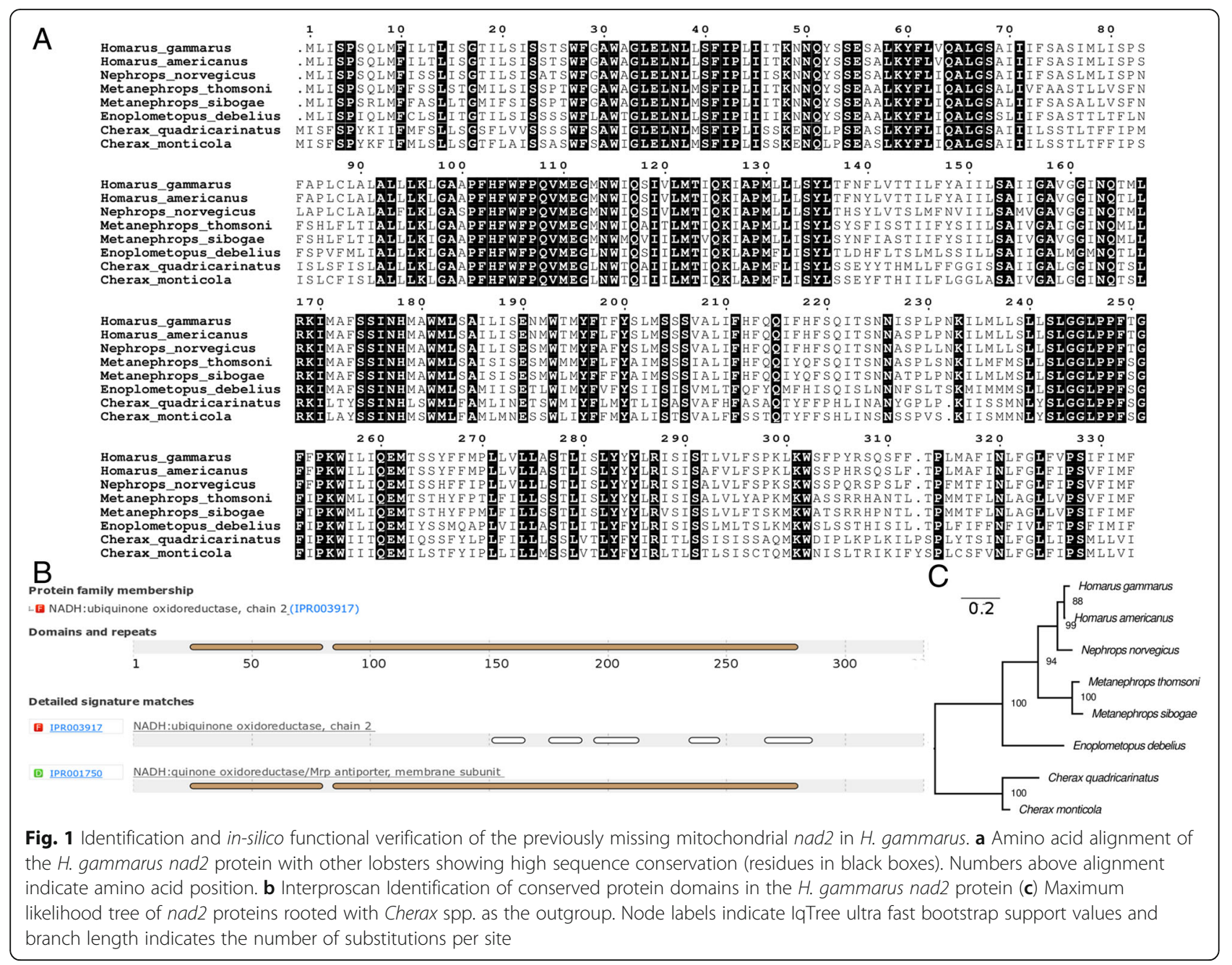

the cox2-atp8-atp6-nad3 protein coding genes, a large inversion and as previously highlighted, the absence of $n a d 2$ and a few tRNAs (Fig. 2a). On average 868 $\mathrm{Mb}(670-1128 \mathrm{Mb})$ of Illumina sequencing data were generated for each of the $H$. gammarus individuals and deposited in the SRA database (Bioproject ID: PRJNA486050). Reference-based mapping assembly successfully reconstructed the near-complete mitogenome except for samples She17_01 and Jer01 that contain minor assembly gaps in the ribosomal genes and non-coding control region (Fig. $2 \mathrm{~b}$ and Additional file 2: Data 1). Notably, significant nucleotide alignment to the $H$. gammarus mitochondrial nad2 gene with high pairwise identity was observed across all five individuals (Fig. 2c).

\section{Different lines of evidence indicate partial tandem duplication in the Homarus gammarus mitogenome} A gene region consisting of $\operatorname{cox} 2$, atp 8 , atp6, cox3, nad3, and nad5 as well as their neighboring tRNAs were present in two copies and in tandem in the newly assembled mitogenome. One of the nad5 genes was substantially shorter with its start codon and a majority of $5^{\prime}$ end truncated (Fig. 3a). The observed duplication was not due to sequencing or assembly error as multiple Nanopore reads spanned across both duplicated gene regions (Fig. 3b). In addition, de novo assembly using Illumina-only reads also recovered two separate contigs corresponding to each of the duplicated region suggesting that sufficient nucleotide dissimilarity exists between the repeats to prevent collapsing of contigs by the assembler into a single contig (Fig. 3c). Substantial and similar coverage of Illumina reads to both regions lends additional support to the veracity of the nanopore sequences. Interestingly, unlike the Nanopore read alignment, Illumina reads show an uneven coverage across the mitogenome that negatively correlates with the genome AT-content (Fig. 3d and e). Notably, low or near-zero read depth was observed in regions with less than $22 \% \mathrm{GC}$ that correspond to the location of the $12 \mathrm{~S}$ and $16 \mathrm{~S}$ ribosomal RNA genes. 

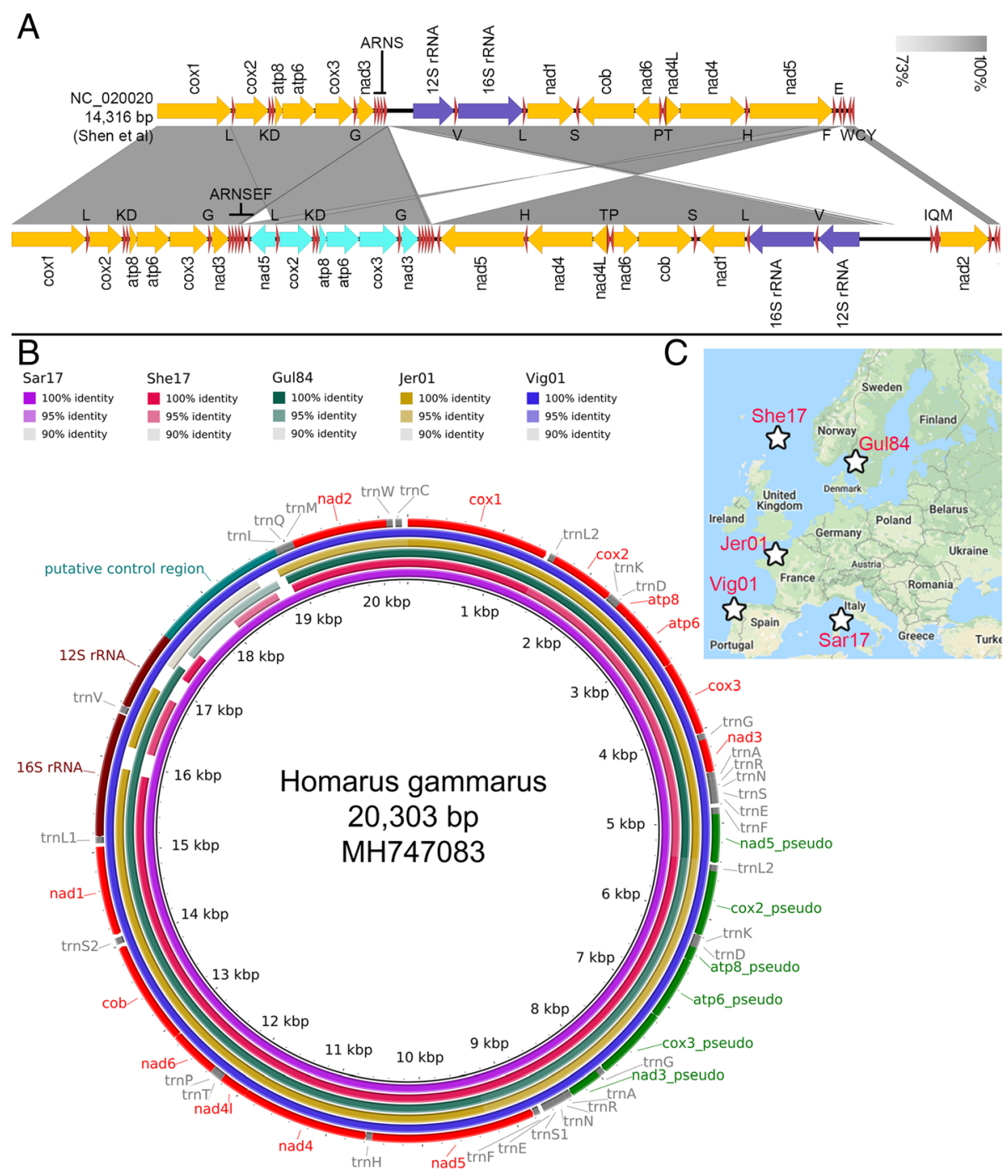

Fig. 2 Presence of nad2 can be detected in at least five H. gammarus individuals collected from various European regions. a Linear gene organization comparison of the previous reported H. gammarus mitogenome with that reported in this study confirming the absence of nad2 in the original report in addition to substantial structural variation. The direction of arrow indicates transcription orientation. Orange, teal, purple and red arrows correspond to protein-coding genes, putative pseudogenes, rRNAs and tRNAs, respectively. $\mathbf{b}$ Circular comparison of the reconstructed mitogenomes showing significant nucleotide alignment across the entire newly assembled $\mathrm{H}$. gammarus mitogenome with minor assembly gaps in the high-AT ribosomal rRNA regions (See Fig. 3e for details). c Sampling map of the fiveH. gammarus individuals with their exact location indicated by a star symbol

\section{Pseudogenization among tandemly duplicated mitochondrial genes}

Duplicated genes that were disconnected from their original transcriptional track e.g. $\operatorname{cox} 2, \operatorname{atp} 8, \operatorname{atp} 6, \operatorname{cox} 3$, and nad3 in the second duplicated region and nad5 in the first duplicated region, appear to show signs of pseudogenization. For example, an internal stop codon was located in the duplicated cox2 gene (Fig. 4a) while frameshift mutations were found in the cox3, nad3, and atp8 genes (Fig. 4b). DNA mutations leading to pseudogenization were supported by multiple Illumina reads, precluding the possibility of assembly or sequencing errors. Although both duplicated atp6 genes are still intact and can be translated into protein sequence of the same length, protein similarity search using the ATPase6 of $H$. americanus as the query against both ATPase6 proteins indicates that the ATPase 6 encoded by the gene located in the first duplicated region is likely the functional one as evidenced by its strikingly high protein identity to that of $H$. americanus $(99.1 \%)$ compared to its duplicated partner (94.6\%). Furthermore, the pseudo ATPase6 exhibits a substantially longer branch length compared to its homologs in the maximum likelihood tree (Fig. 4c), suggesting higher substitution rate presumably due to the lack of evolutionary constraints. It is also interesting to note that the pseudo ATPase6 clustered with the 


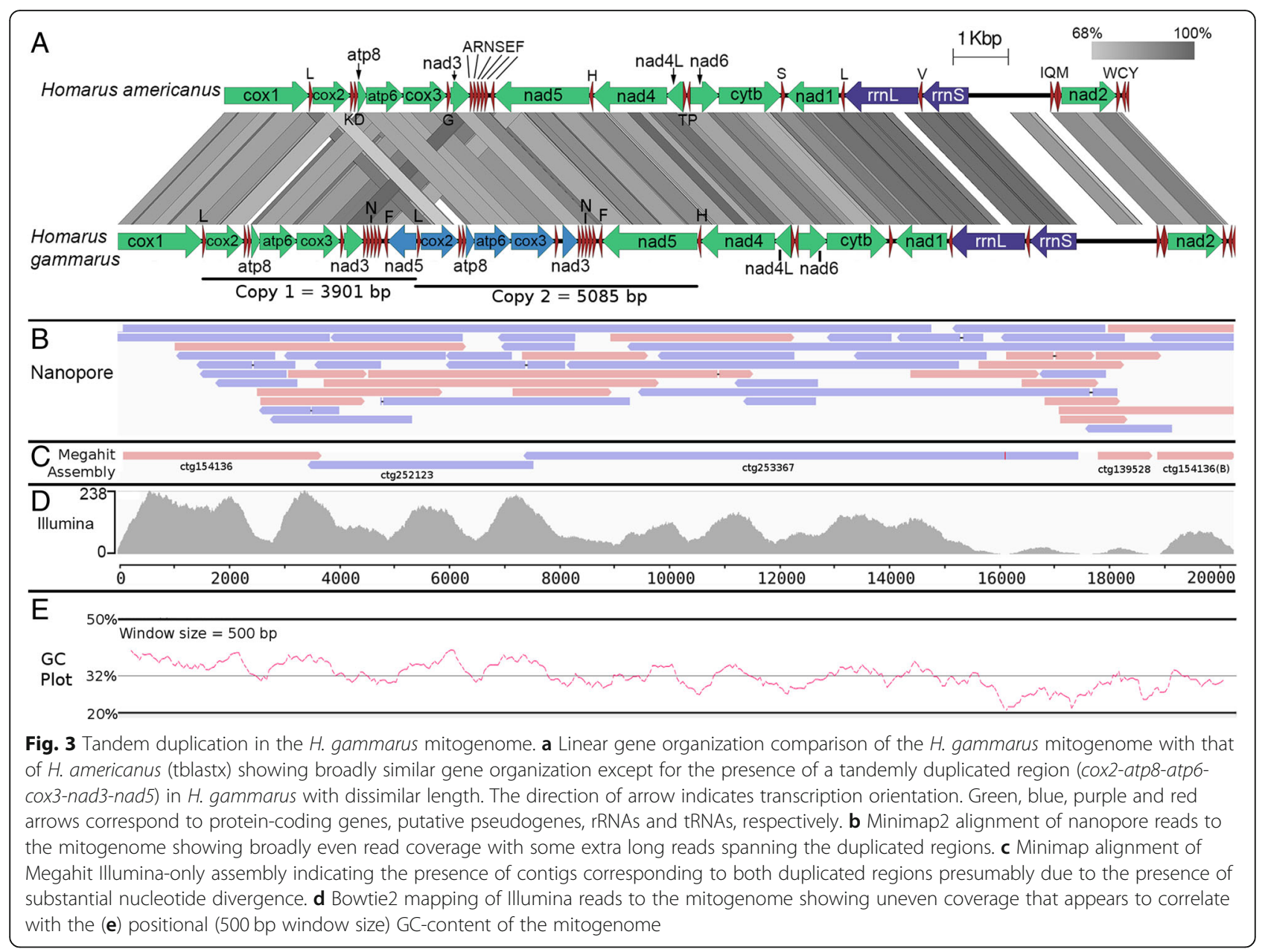

previously published Homarus gammarus ATPase6 that also shows a similar branch length pattern.

\section{Discussion}

The veracity of the current European lobster reference mitogenome in the NCBI database (Accession Number: NC_020020.1) is questioned given its unusual features e.g. missing nad2, large mitogenome inversion and translocated tRNAs $[9,10,24]$ that were not observed in its close relative, the American lobster, Homarus americanus [8]. Although subsequent PCR and Sanger sequencing has been carried out on additional individuals to validate the observed abnormalities [10], a secondary validation by a separate group using different sequencing technology is highly desirable to provide confirmation of this atypical crustacean mitogenome sequence. We, therefore, sequenced another individual of Homarus gammarus using Illumina technology, which removes the need for the laborious and presumably error-prone steps required in traditional mitogenome sequencing such as long-range PCR and primer walking that may lead to the unintentional misassembly of mitogenome.
The absence of a repetitive region and/or duplicated gene region in the previously reported $H$. gammarus mitogenome [9] (Fig. 2a) suggests that it should be possible to recover a complete mitogenome using Illumina short reads as demonstrated in various crustacean mitogenome reports [16, 21, 22, 56, 57]. However, none of our attempted Illumina-only assemblies recovered a circular mitogenome, indicating the potential presence of a problematic region in the mitogenome that could not be resolved, presumably due to the limitation of short reads in recovering regions with long repeats. Only by leveraging on the availability of Oxford Nanopore long-read technology, were we able to confidently close this mitogenome. In doing so, we uncovered the presence of an unforeseen duplicated region in the H. gammarus mitogenome in addition to the presence of the nad2 gene which was previously reported as missing [9]. The identification of multiple Nanopore reads spanning the entire duplicated region lends support to the authenticity of the duplication observed in the mitogenome assembly. Furthermore, by employing the Nanopore PCR-free approach to sequencing e.g. by directly sequencing the native DNA, 


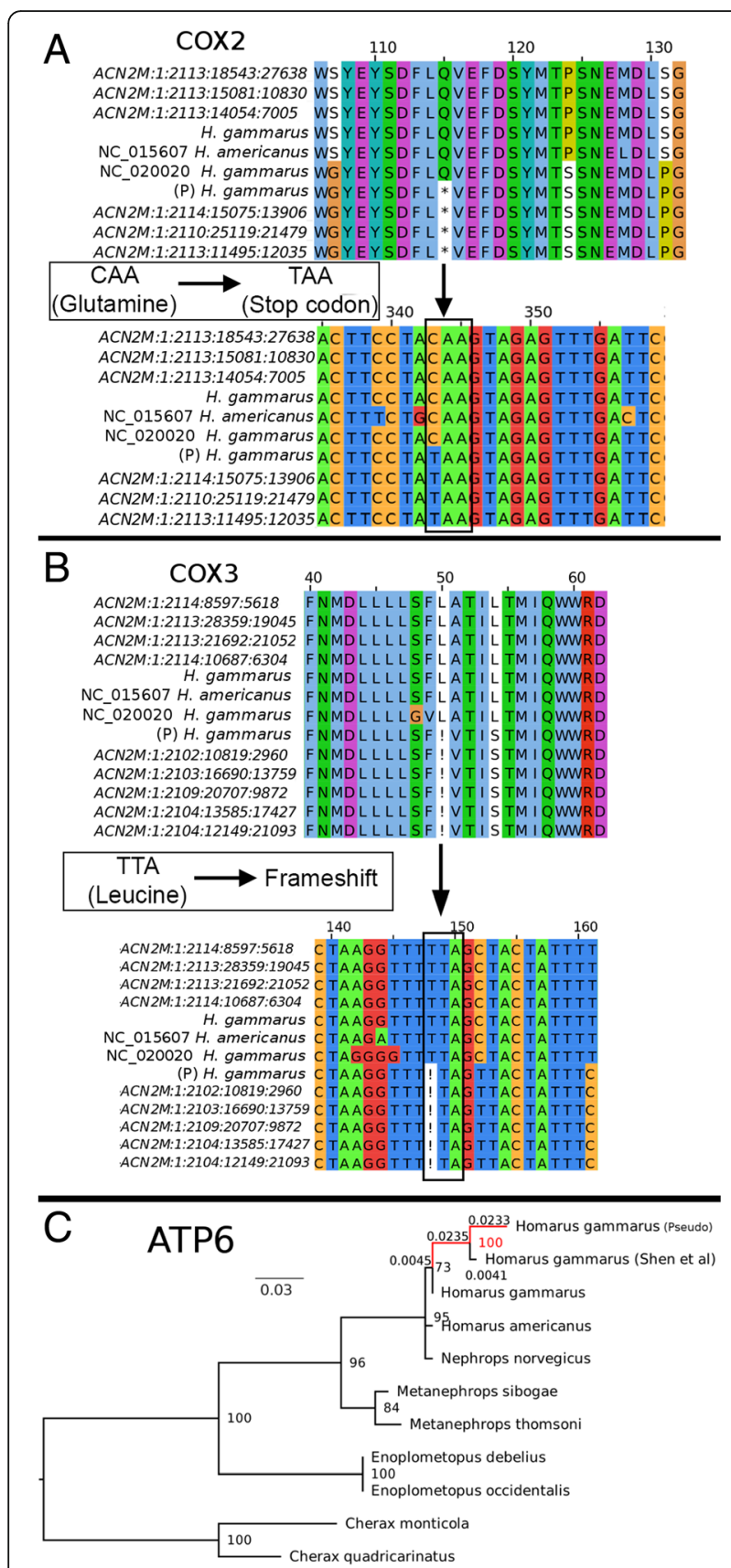

Fig. 4 Evidence for pseudogenization among genes located in the duplicated regions. Identification of (a) Internal stop codon and (b) Frameshift mutation in one of the duplicated cox 2 and cox3 genes, respectively, with strong support from multiple Illumina short reads. Labels with bracketed "P" indicate putative pseudogenes (c) Maximum likelihood tree constructed based on the protein alignment of ATP6. Labels above branches are branch lengths representing the number of amino acid substitutions per site

we can safely disregard misassembly due to chimeric sequences from PCR-amplified products commonly associated with long-range PCR $[58,59]$. It is also fortuitous that the duplicated regions have accumulated sufficient mutation resulting in the assembly of both regions as separate contigs using Illumina-only reads which provides additional and complementary support for the presence of tandem duplication in the H. gammarus mitogenome recovered from the Nanopore reads. The length of the observed large inversion between Shen et al [9] H. gammarus assembly (GenBank: NC_020020) and ours (GenBank: MH747083) (Fig. 2a) is consistent with the estimated amplicon length generated by the primers used for long-range PCR raising the possibility that misassembly could occur during the scaffolding of the assembled amplicons.

The clustering of the published $H$. gammarus AT6ase with the pseudo-ATP6ase recovered in this study coupled with its notably longer branch length compared to the bona fide $H$. gammarus, H. americanus, and Nephrops norvegicus ATP6ases strongly suggests that this atp6 gene is a chimera of the bona fide and pseudogenes. The use of long-range PCR can result in the amplification of both duplicated region that will be considered as a single PCR product given their near-identical amplicon size. Subsequent Sanger sequencing of the "mixed" amplicons will result in the presence of non-random mixed peaks in the sequencing chromatogram that will likely be haphazardly regarded as heteroplasmy. Depending on the peak intensity, alleles originating from the other duplicated region may be called leading to the assembly of a chimeric sequence.

Based on an initial analysis of $100 \mathrm{H}$. gammarus individuals, Triantafyllidis et al. used restriction fragment length polymorphism (RFLP) profiling to show that a $3 \mathrm{~kb}$ mitochondrial region (amplified using the Hom3F and Hom3R primers) containing cox2, cox3, atp6, atp8, nad3 and several tRNAs is the most variable region and hence selected that region for a population genetic study of 4018 individuals collected throughout the geographical range of the species [7]. This is quite surprising given that the $3 \mathrm{~kb}$ region does not contain the control region which is known to be the most polymorphic region in mitochondrial DNA [60], suggesting an unknown source of nucleotide variation in addition to population-specific genetic variation. Similarity search of Hom3F and Hom3R primers against the newly assembled Homarus gammarus mitogenome indicates that primer binding sites are present in both of the duplicated regions which will lead to the amplification of two amplicons with near exact length $(\sim 2.8 \mathrm{~kb})$ (Additional file 3 : Table S1), and may well have confounded the results of Triantafyllidis et al. [7].

Mapping of Illumina reads to the mitogenome revealed a GC-dependent coverage variation that was not observed in the Nanopore read alignment. Since the standard Nanopore library preparation does not involve any PCR step, the uneven mapping coverage is most definitely caused by PCR amplification bias during Illumina library preparation which is known to under-represent AT-rich fragments $[61,62]$. AT-rich regions are prevalent in the 
control region and ribosomal RNA genes. Such mitogenome regions will be harder to assemble due to low sequencing depth, leading to gaps in the mitogenome assembly and the incorrect inference of mitogenome linearity. Although our study highlights the value of Nanopore long-read technology in resolving a difficult-to-assemble mitogenome, we are conservative in advocating its use in routine mitogenome sequencing due to its stringent requirement for high-quality and intact gDNA. Having said that, the obvious advantage that Nanopore long reads offer in spanning repetitive sequencing make it a powerful new tool for elucidating or confirming duplicated mitochondrial genes and difficult-to-assemble mitogenomes in animals. Another third generations sequencing approach that can be used to improve a mitogenome assembly is PacBio technology albeit its reliance on polymerase chain reaction to generate sequencing data may reduce its efficiency in the sequencing of problematic region, as encountered in Illumina sequencing in this study.

Lastly, we emphasize as reported in other scientific domains, that the absence of evidence (for a mitochondrial gene) is not necessary evidence of absence. A gene may fail to be recovered due to limitation in a wet lab protocol or bioinformatics pipeline as we demonstrated for the nad2 gene in $H$. gammarus in this study and others have demonstrated for the atp 8 gene in bivalve mollusks [63]. Thus, researchers need to be skeptical of assertions that a mitochondrial gene is missing and make efforts to verify that there really is a genuine evidence of absence.

\section{Conclusions}

We demonstrate the utility of Oxford Nanopore long-read in resolving problematic mitogenome assemblies by re-sequencing the mitogenome of the European lobster that was previously reported to exhibit several atypical features. In addition to showing substantial deviation from the previously published mitogenome, we identified new gene features that were missed by previous studies including the complete recovery of the nad2 gene and a large tandem duplication consisting of six protein-coding genes with evidence of pseudogenization. The power of long reads in resolving repetitive regions and accurately identifying tandemly duplicated mitochondrial gene regions highlights the value of new sequencing tools for evolutionary biologists to confidently infer novel mitochondrial gene organization. Furthermore, the veracity of unusual mitochondrial gene rearrangements recovered using first and second sequencing technologies, particularly those used to make phylogenetic inferences of evolutionary scenarios, can now be effectively tested using Oxford Nanopore long reads, marking another step forward in mitogenomics.

\section{Additional files}

Additional file 1: Figure S1. Long-read only assembly enables the complete assembly of $\mathrm{H}$. gammarus mitogenome. (A) Bandage visualization of the CANU assembly graph corresponding to the mitogenome contig. (B) Self-against-self comparison of the mitogenome contig in EasyFig with up to $6 \mathrm{~kb}$ of flanking regions exhibiting high nucleotide similarity ( 98\%) (TIF 76 kb)

Additional file 2: Data 1. Mitogenome assemblies in fasta format of 5 additional Homarus gammarus sampled from distant location in Europe (See Fig. 2c for details) (TXT 99 kb)

Additional file 3: Table S1. Binding sites of I_Hom3F and I_Hom3R primers on the newly assembled Homarus gammarus mitogenome and the estimated PCR product sizes. (PDF $16 \mathrm{~kb}$ )

\section{Abbreviations}

PCG: Protein Coding Gene; PCR: Polymerase Chain Reaction; SDS: Sodium Dodecyl Sulfate; SNP: Single Nucleotide Polymorphism

\section{Acknowledgements}

Not applicable.

\section{Funding}

Funding for this project was provided by the Monash University Malaysia Tropical Medicine and Biology Platform. The funder had no role in the design of the study and collection, analysis, and interpretation of data and in writing the manuscript.

\section{Availability of data and materials}

Illumina and Nanopore sequencing data are available through the NCBI SRA accession SRP157954 and Zenodo repository (https://doi.org/10.5281/ zenodo.1345356), respectively.

\section{Authors' contributions}

HMG, CMA, TL and FG designed and coordinated the research. FG and TL collected the sample. HMG performed sequencing and bioinformatics analysis. HMG, CMA and FG analyzed the data and drafted the manuscript. All authors read and approved the final manuscript.

Ethics approval and consent to participate

Not applicable. Samples (deceased) were either purchased from or given by commercial fisherman.

Consent for publication

Not applicable.

\section{Competing interests}

The authors declare that they have no competing interests.

\section{Publisher's Note}

Springer Nature remains neutral with regard to jurisdictional claims in published maps and institutional affiliations.

\section{Author details}

${ }^{1}$ Deakin Genomics Centre, Deakin University, Geelong, VIC 3220, Australia. ${ }^{2}$ Centre for Integrative Ecology, School of Life and Environmental Sciences, Deakin University, Geelong, VIC 3220, Australia. ${ }^{3}$ School of Science, Monash University Malaysia, Bandar Sunway, 47500 Petaling Jaya, Malaysia. ${ }^{4}$ Monash University Malaysia Genomics Facility, Monash University, Bandar Sunway, 47500 Petaling Jaya, Malaysia. ${ }^{5}$ Laboratoire Ecologie et Biologie des Interactions, UMR CNRS 7267 Equipe Ecologie Evolution Symbiose 5 rue Albert Turpin, 86073 Poitiers, Cedex, France. 'Department of Biosciences, College of Life and Environmental Sciences, University of Exeter, Exeter, UK. 


\section{Received: 26 August 2018 Accepted: 16 April 2019}

\section{Published online: 03 May 2019}

\section{References}

1. Galparsoro I, Borja Á, Bald J, Liria P, Chust G. Predicting suitable habitat for the European lobster (Homarus gammarus), on the Basque continental shelf (Bay of Biscay), using ecological-niche factor analysis. Ecol Model. 2009; 220(4):556-67.

2. Kenchington EL, Harding GC, Jones MW, Prodoehl PA. Pleistocene glaciation events shape genetic structure across the range of the American lobster, Homarus americanus. Mol Ecol. 2009;18(8):1654-67.

3. Tracey ML, Nelson K, Hedgecock D, Shleser RA, Pressick ML. Biochemical genetics of lobsters: genetic variation and the structure of American lobster (Homarus americanus) populations. J Fisheries Board Canada. 1975;32(11): 2091-101.

4. Jones MW, O'Reilly PT, McPherson AA, McParland TL, Armstrong DE, Cox AJ, Spence KR, Kenchington EL, Taggart CT, Bentzen P. Development, characterisation, inheritance, and cross-species utility of American lobster (Homarus americanus) microsatellite and mtDNA PCR-RFLP markers. Genome. 2003;46(1):59-69.

5. Ulrich I, Müller J, Schütt C, Buchholz F. A study of population genetics in the European lobster, Homarus gammarus (Decapoda, Nephropidae). Crustaceana. 2001;74(9):825-37.

6. Jørstad KE, Prodohl P, Agnalt A-L, Hughes M, Farestveit E, Ferguson A Comparison of genetic and morphological methods to detect the presence of American lobsters, Homarus americanus H. Milne Edwards, 1837 (Astacidea: Nephropidae) in Norwegian waters. Hydrobiologia. 2007;590(1):103-14.

7. Triantafyllidis A, Apostolidis A, Katsares V, Kelly E, Mercer J, Hughes M, Jørstad K, Tsolou A, Hynes R, Triantaphyllidis C. Mitochondrial DNA variation in the European lobster (Homarus gammarus) throughout the range. Mar Biol. 2005;146(2):223-35.

8. Kim S, Lee S-H, Park M-H, Choi H-G, Park J-K, Min G-S. The complete mitochondrial genome of the American lobster, Homarus americanus (Crustacea, Decapoda). Mitochondrial DNA. 2011;22(3):47-9.

9. Shen H, Braband A, Scholtz G. Mitogenomic analysis of decapod crustacean phylogeny corroborates traditional views on their relationships. Mol Phylogenet Evol. 2013;66(3):776-89.

10. Shen H, Braband A, Scholtz G. The complete mitogenomes of lobsters and crayfish (Crustacea: Decapoda: Astacidea) reveal surprising differences in closely related taxa and convergences to Priapulida. J Zool Syst Evol Res. 2015;53(4):273-81.

11. Gan HM, Tan MH, Eprilurahman R, Austin CM. The complete mitogenome of Cherax monticola (Crustacea: Decapoda: Parastacidae), a large highland crayfish from New Guinea. Mitochondrial DNA Part A. 2016;27(1):337-8.

12. Gan HM, Tan MH, Austin CM. The complete mitogenome of the red claw crayfish Cherax quadricarinatus (Von martens, 1868)(Crustacea: Decapoda: Parastacidae). Mitochondrial DNA Part A. 2016;27(1):385-6.

13. Austin CM, Tan MH, Gan HY, Gan HM. The complete mitogenome of the endangered freshwater crayfish Cherax tenuimanus (smith 1912)(Crustacea: Decapoda: Parastacidae). Mitochondrial DNA Part A. 2016;27(6):4176-7.

14. Gan HM, Tan MH, Lee YP, Schultz MB, Horwitz P, Burnham Q, Austin CM. More evolution underground: accelerated mitochondrial substitution rate in Australian burrowing freshwater crayfishes (Decapoda: Parastacidae). Mol Phylogenet Evol. 2018;118:88-98.

15. Ahn D-H, Min G-S, Park J-K, Kim S. The complete mitochondrial genome of the red-banded lobster Metanephrops thomsoni (Crustacea, Astacidea, Nephropidae): a novel gene order. Mitochondrial DNA Part A. 2016;27(4):2663-4.

16. Tan MH, Gan HM, Schultz MB, Austin CM. MitoPhAST, a new automated mitogenomic phylogeny tool in the post-genomic era with a case study of 89 decapod mitogenomes including eight new freshwater crayfish mitogenomes. Mol Phylogenet Evol. 2015;85:180-8.

17. Nguyen TT, Murphy NP, Austin CM. Amplification of multiple copies of mitochondrial cytochrome b gene fragments in the Australian freshwater crayfish, Cherax destructor Clark (Parastacidae: Decapoda). Anim Genet. 2002; 33(4):304-8

18. Miller AD, Nguyen TT, Burridge CP, Austin CM. Complete mitochondrial DNA sequence of the Australian freshwater crayfish, Cherax destructor (Crustacea: Decapoda: Parastacidae): a novel gene order revealed. Gene. 2004;331:65-72.

19. Bensasson D, Zhang D-X, Hartl DL, Hewitt GM. Mitochondrial pseudogenes: evolution's misplaced witnesses. Trends Ecol Evol. 2001;16(6):314-21.
20. Gan HM, Schultz MB, Austin CM. Integrated shotgun sequencing and bioinformatics pipeline allows ultra-fast mitogenome recovery and confirms substantial gene rearrangements in Australian freshwater crayfishes. BMC Evol Biol. 2014;14(1):19.

21. Tan MH, Gan HM, Lee YP, Linton S, Grandjean F, Bartholomei-santos ML, Miller AD, Austin CM. ORDER within the chaos: insights into phylogenetic relationships within the Anomura (Crustacea: Decapoda) from mitochondrial sequences and gene order rearrangements. Mol Phylogenet Evol. 2018;127:320-31.

22. Tan MH, Gan HM, Dally G, Horner S, Moreno PAR, Rahman S, Austin CM. More limbs on the tree: mitogenome characterisation and systematic position of IIiving fossil'species Neoglyphea inopinata and Laurentaeglyphea neocaledonica (Decapoda: Glypheidea: Glypheidae). Invertebr Syst. 2018;32(2):448-56.

23. Paijmans JL, Gilbert MTP, Hofreiter M. Mitogenomic analyses from ancient DNA. Mol Phylogenet Evol. 2013;69(2):404-16.

24. Gan HM, Tan MH, Gan HY, Lee YP, Austin CM. The complete mitogenome of the Norway lobster Nephrops norvegicus (Linnaeus, 1758)(Crustacea: Decapoda: Nephropidae). Mitochondrial DNA Part A. 2016;27(5):3179-80.

25. Grandjean F, Tan MH, Gan HM, Lee YP, Kawai T, Distefano RJ, Blaha M, Roles AJ, Austin CM. Rapid recovery of nuclear and mitochondrial genes by genome skimming from northern hemisphere freshwater crayfish. Zool Scr. 2017;46(6):718-28.

26. Rang FJ, Kloosterman WP, de Ridder J. From squiggle to basepair: computational approaches for improving nanopore sequencing read accuracy. Genome Biol. 2018;19(1):90.

27. Tan MH, Austin CM, Hammer MP, Lee YP, Croft LJ, Gan HM. Finding nemo: hybrid assembly with Oxford Nanopore and Illumina reads greatly improves the clownfish (Amphiprion ocellaris) genome assembly. GigaScience. 2018; 7(3):gix137.

28. Austin CM, Tan MH, Harrisson KA, Lee YP, Croft LJ, Sunnucks P, Pavlova A, Gan HM. De novo genome assembly and annotation of Australia's largest freshwater fish, the Murray cod (Maccullochella peelii), from Illumina and Nanopore sequencing read. GigaScience. 2017;6(8):1-6.

29. Gan HM, Lee YP, Austin CM. Nanopore long-read guided complete genome assembly of Hydrogenophaga intermedia, and genomic insights into 4aminobenzenesulfonate, $\mathrm{p}$-aminobenzoic acid and hydrogen metabolism in the genus Hydrogenophaga. Front Microbiol. 2017:8:1880.

30. Ranjard L, Wong TK, Külheim C, Rodrigo AG, Ragg NL, Patel S, Dunphy BJ. Complete mitochondrial genome of the green-lipped mussel, Perna canaliculus (Mollusca: Mytiloidea), from long nanopore sequencing reads. Mitochondrial DNA Part B. 2018;3(1):175-6.

31. Froufe E, Gan HM, Lee YP, Carneiro J, Varandas S, Teixeira A, Zieritz A, Sousa $\mathrm{R}$, Lopes-Lima M. The male and female complete mitochondrial genome sequences of the endangered freshwater mussel Potomida littoralis (Cuvier, 1798)(Bivalvia: Unionidae). Mitochondrial DNA Part A. 2016;27(5):3571-2.

32. Lopes-Lima M, Fonseca MM, Aldridge DC, Bogan AE, Gan HM, Ghamizi M, Sousa R, Teixeira A, Varandas S, Zanatta D. The first Margaritiferidae male (M-type) mitogenome: mitochondrial gene order as a potential character for determining higher-order phylogeny within Unionida (Bivalvia). J Molluscan Stud. 2017;83(2):249-52.

33. Burzyński A, Soroka M, Mioduchowska M, Kaczmarczyk A, Sell J. The complete maternal and paternal mitochondrial genomes of Unio crassus: mitochondrial molecular clock and the overconfidence of molecular dating. Mol Phylogenet Evol. 2017;107:605-8.

34. Lopes-Lima M, Bolotov IN, Aldridge DC, Fonseca MM, Gan HM, Gofarov MY, Kondakov AV, Prié V, Sousa R, Varandas S. Expansion and systematics redefinition of the most threatened freshwater mussel family, the Margaritiferidae. Mol Phylogenet Evol. 2018;127:98-118.

35. Sokolov EP. An improved method for DNA isolation from mucopolysacchariderich molluscan tissues. J Molluscan Stud. 2000;66(4):573-5.

36. Jenkins TL, Ellis CD, Stevens JR. SNP discovery in European lobster (Homarus gammarus) using RAD sequencing. Conserv Genet Resour. 2018. p. 1-5. https://doi.org/10.1007/s12686-018-1001-8.

37. Li D, Liu C-M, Luo R, Sadakane K, Lam T-W. MEGAHIT: an ultra-fast singlenode solution for large and complex metagenomics assembly via succinct de Bruijn graph. Bioinformatics. 2015;31(10):1674-6.

38. Dierckxsens N, Mardulyn P, Smits G. NOVOPlasty: de novo assembly of organelle genomes from whole genome data. Nucleic Acids Res. 2016;45(4):e18.

39. Hahn C, Bachmann L, Chevreux B. Reconstructing mitochondrial genomes directly from genomic next-generation sequencing reads-a baiting and iterative mapping approach. Nucleic Acids Res. 2013;41(13):e129. 
40. Koren S, Walenz BP, Berlin K, Miller JR, Bergman NH, Phillippy AM. Canu: scalable and accurate long-read assembly via adaptive $k$-mer weighting and repeat separation. Genome Res. 2017;27(5):722-36. https://doi.org/10.1101/ gr.215087.116.

41. Wick RR, Schultz MB, Zobel J, Holt KE. Bandage: interactive visualization of de novo genome assemblies. Bioinformatics. 2015;31 (20):3350-2.

42. Sullivan MJ, Petty NK, Beatson SA. Easyfig: a genome comparison visualizer. Bioinformatics. 2011;27(7):1009-10.

43. Wick RR, Judd LM, Gorrie CL, Holt KE. Unicycler: resolving bacterial genome assemblies from short and long sequencing reads. PLoS Comput Biol. 2017; 13(6):e1005595.

44. Langmead B, Salzberg SL. Fast gapped-read alignment with bowtie 2. Nat Methods. 2012;9(4):357.

45. Walker BJ, Abeel T, Shea T, Priest M, Abouelliel A, Sakthikumar S, Cuomo CA, Zeng Q, Wortman J, Young SK. Pilon: an integrated tool for comprehensive microbial variant detection and genome assembly improvement. PLoS One. 2014;9(11):e112963.

46. Bernt $M$, Donath A, Jühling F, Externbrink F, Florentz C, Fritzsch G, Pütz J, Middendorf M, Stadler PF. MITOS: improved de novo metazoan mitochondrial genome annotation. Mol Phylogenet Evol. 2013;69(2):313-9.

47. Katoh K, Standley DM. MAFFT multiple sequence alignment software version 7: improvements in performance and usability. Mol Biol Evol. 2013; 30(4):772-80.

48. Gouet P, Robert $X$, Courcelle E. ESPript/ENDscript: extracting and rendering sequence and 3D information from atomic structures of proteins. Nucleic Acids Res. 2003;31(13):3320-3.

49. Nguyen L-T, Schmidt HA, von Haeseler A, Minh BQ. IQ-TREE: a fast and effective stochastic algorithm for estimating maximum-likelihood phylogenies. Mol Biol Evol. 2014;32(1):268-74.

50. Alikhan NF, Petty NK, Ben Zakour NL, Beatson SA. BLAST ring image generator (BRIG): simple prokaryote genome comparisons. BMC Genomics. 2011;12:402

51. Thorvaldsdóttir H, Robinson JT, Mesirov JP. Integrative genomics viewer (IGV): high-performance genomics data visualization and exploration. Brief Bioinform. 2013;14(2):178-92.

52. Li H. Minimap2: pairwise alignment for nucleotide sequences. Bioinformatics. 2018;1:7.

53. Rutherford K, Parkhill J, Crook J, Horsnell T, Rice P, Rajandream M-A, Barrell B. Artemis: sequence visualization and annotation. Bioinformatics. 2000; 16(10):944-5.

54. Ranwez V, Harispe S, Delsuc F, Douzery EJ. MACSE: multiple alignment of coding SEquences accounting for frameshifts and stop codons. PLoS One. 2011;6(9):e22594.

55. Waterhouse AM, Procter JB, Martin DM, Clamp M, Barton GJ. Jalview version 2-a multiple sequence alignment editor and analysis workbench. Bioinformatics. 2009;25(9):1189-91.

56. Tan MH, Gan HM, Lee YP, Poore GC, Austin CM. Digging deeper: new gene order rearrangements and distinct patterns of codons usage in mitochondrial genomes among shrimps from the Axiidea, Gebiidea and Caridea (Crustacea: Decapoda). PeerJ. 2017;5:e2982.

57. Gan HM, Tan MH, Austin CM. The complete mitogenome of the Australian spiny crayfish Euastacus yarraensis (McCoy, 1888) (Crustacea: Decapoda: Parastacidae). Mitochondrial DNA Part A. 2016;27(2):929-30.

58. Haas BJ, Gevers D, Earl AM, Feldgarden M, Ward DV, Giannoukos G, Ciulla D, Tabbaa D, Highlander SK, Sodergren E. Chimeric 165 rRNA sequence formation and detection in sanger and 454-pyrosequenced PCR amplicons. Genome Res. 2011;21(3):494-504. https://doi.org/10.1101/gr.112730.110.

59. Kalle E, Kubista M, Rensing C. Multi-template polymerase chain reaction. Biomol Detect Quantification. 2014;2:11-29.

60. Stoneking M. Hypervariable sites in the mtDNA control region are mutational hotspots. Am J Hum Genet. 2000;67(4):1029-32.

61. Aird D, Ross MG, Chen W-S, Danielsson M, Fennell T, Russ C, Jaffe DB, Nusbaum C, Gnirke A. Analyzing and minimizing PCR amplification bias in Illumina sequencing libraries. Genome Biol. 2011;12(2):R18.

62. Oyola SO, Otto TD, Gu Y, Maslen G, Manske M, Campino S, Turner DJ, Maclnnis B, Kwiatkowski DP, Swerdlow HP. Optimizing Illumina nextgeneration sequencing library preparation for extremely AT-biased genomes. BMC Genomics. 2012;13(1):1.

63. Lubośny M, Przyłucka A, Śmietanka B, Breton S, Burzyński A. Actively transcribed and expressed atp8 gene in Mytilus edulis mussels. PeerJ. 2018;6: e4897.

\section{Ready to submit your research? Choose BMC and benefit from}

- fast, convenient online submission

- thorough peer review by experienced researchers in your field

- rapid publication on acceptance

- support for research data, including large and complex data types

- gold Open Access which fosters wider collaboration and increased citations

- maximum visibility for your research: over $100 \mathrm{M}$ website views per year

At BMC, research is always in progress.

Learn more biomedcentral.com/submissions 